\title{
Experience of Persistent Gestational Trophoblastic Disease in a Tertiary Medical College Hospital, Bangladesh
}

\author{
NAHAR KAMRUN ${ }^{1}$, YESMIN HALIMA $^{2}$, ROY KANIKA ${ }^{3}$, ALAM SAFIUL ${ }^{4}$, KHATUN KASHEFA $^{5}$
}

\begin{abstract}
:
Objectives: To study the clinical presentation and risk factors of persistent trophoblastic disease and its outcome of treatment with chemotherapy.

Materials and methods: This observational study was carried out on fifty patients of persistent trophoblastic disease who were admitted in the Department of Obstetrics \& Gynaecology, Mymensingh Medical Hospital (MMCH) during one year period. Evaluation of disease was done by thorough clinical examination and a set of investigations including chest radiography, ultrasound scan of abdomen and pelvis and estimation of serum â hCG. The four factors under analysis of PTD were age of the patient, clinical presentation, gestational age at diagnosis of molar pregnancy and nature of antecedent pregnancy.
\end{abstract}

Patients with non-metastatic trophoblastic disease and low risk metastatic trophoblastic disease were offered single agent chemotherapy with methotrexate and folinic acid rescue in consultation with the oncologist. When $\beta$ hCG response was inadequate, multi-agent chemotherapy was given. Complications of chemotherapy were also observed and supportive treatment was given.

Results: Out of 50 patients, 49 (98\%) patients had antecedent molar pregnancy and 1(2\%) had missed abortion. In cases of post molar trophoblastic disease, 28 (57.58\%) were in $20-30 \mathrm{yrs}$. Mean $\pm S D$ was $31.35 \pm 7.25$. In these cases gestational size of molar pregnancy was between 16-20 weeks in 24 (48.98\%), <16 wks in 19 (38.78\%) and >20 wks in 6 $(12.24 \%)$ cases. Mean $\pm S D$ was $16.78 \pm 4.45$ wks $(p<0.001)$. Associated theca lutein cysts were present among higher number of cases (57.14\%) but not statistically significant. Regarding clinical presentation, 40 (80\%) patients presented with irregular pervaginal bleeding, 3(6\%) patients with features of metastasis. Most of the of the study subjects 43 (86\%) were treated with chemotherapy and 7 (14\%) had undergone both hysterectomy and chemotherapy. Single agent methotrexate was given in 47 (94\%) cases and multiple agent (EMA-CO) in 3 (6\%). After giving 4 cycles of chemotherapy 11 (22\%) patients were cured, 38 (76\%) had declining â hCG level and one had static $\beta$ hCG level. Overall remission was $98 \%$. Complication of chemotherapy was observed in 5 (10\%) patients.

Conclusion: Theca lutein cyst are important in the prediction of persistent disease after molar pregnancy. Methotrexate chemotherapy is effective and well tolerated in treating patients with nonmetastatic and low risk metastatic gestational trophoblastic neoplasia.

Key Words: Persistent trophobtastic disease, risk factor, chemotherapy.

\section{Introduction:}

Gestational trophoblastic disease is a spectrum of tumors with a wide range of biologic behaviour and potential for metastasis. It refers to both the benign and malignant entities of the spectrum and includes hydatidiform mole, invasive mole, choriocarcinoma and placental site trophoblastic tumour (PSTT). The last three are termed gestational trophoblastic tumours

1. Professor, Dept. of Obs. \& Gynae, Green Life Medical College, Dhaka.

2. Medical Officer, Fulpur Thana Health Complex, Mymensingh.

3. Medical Officer, Dept. of Obs. \& Gynae, Mymensingh Medical College, Mymensingh.

4. Associate Professor, Dept. of Radiotherapy, Mymensingh Medical College, Mymensingh.

5. Assistant Professor, Dept. of Obs. \& Gynae, Mymensingh Medical College, Mymensingh. 
(GTT); all may metastasize and are potentially fatal if untreated ${ }^{1}$. The incidence of hydatidiform mole varies in different regions of the world, but now has been falling ${ }^{1}$. Highest incidence of 12.1/1000 deliveries was reported from Turkey. The malignant potential of this disease is higher in South East Asia where it can be as high as $10-15 \%$ in comparison to $2-4 \%$ in the western countries ${ }^{2}$.

In persistent trophoblastic disease (PTD), trophoblastic activity remains after evacuation of hydatidiform mole as shown by subsequent high or even rising $\beta$ hCG concentration in blood. The reported frequency of PTD is $20 \%$ in complete hydatidiform mole and 0.5 to $9.9 \%$ in partial hydatidiform mole. In order to prevent complication from metastatic disease, PTD needs to be treated early. Prophylactic chemotherapy (started immediately after evacuation of the mole) reduces the incidence of PTD to $4-12 \%{ }^{1}$. Because of the large proportion of patients show spontaneous remission of molar pregnancy after evacuation and because of the side effects of chemotherapy, clinicians are reluctant to use prophylactic chemotherapy. It would therefore be helpful to identify the patients at risk for developing PTD ${ }^{3}$.

Various risk factors for post molar trophoblastic disease has been reported such as pre evacuation $\beta$ hCG level, maternal age, gestational age, histologic grade of molar tissue, uterine size, ovarian cyst, presence of medical complication, previous molar pregnancy and $\mathrm{ABO}$ blood groups were evaluated ${ }^{4}$.

Gestational trophoblastic disease can occur after any type of antecedent pregnancy. The most frequent antecedent pregnancy is that of a patient presenting with a hydatidiform mole. Nearly $45 \%$ of choriocarcinomas follow evacuation of hydatidiform mole, $25 \%$ follow after full term normal pregnancy, 25\% follow spontaneous abortion and $5 \%$ ectopic pregnancy ${ }^{5}$.

The leading symptom of persistent trophoblastic disease is irregular uterine haemorrhage coming sooner or later after the expulsion of a mole or a normal pregnancy. Often the disease presents by way of its metastasis. Thus the occurrence of a haemothorax, a complaint of dyspnoea or haemoptysis or the appearance of neurological signs and symptoms such as headache, visual disturbances or focal neurological deficits can be the first evidence of choriocarcinoma ${ }^{6}$.
Patients with gestational trophoblastic tumors are stratified into low and high risk groups which have different treatments and outcomes. A number of factors are used to stratify patients into high and low risk groups. Each factor scores numerical points. A score $>6$ (FIGO score) or >8 (Charring cross score) defines high risk disease ${ }^{7}$. Low risk patients are usually treated with single agent chemotherapy. Approximately $85-90 \%$ of patients in this group are cured by the initial chemotherapy regimen. Most of the others respond to alternate drugs; combination chemotherapy is rarely needed. High risk patients require combination chemotherapy with a selective use of surgery and radiotherapy ${ }^{1}$.

Since there are well known side effects of all the chemotherapeutic drugs, a careful check is made before a course of treatment is commenced or continued. The principal adverse effects are on the bone marrow, liver and kidney, so regular checks are made on white cell count, platelets, liver function and blood urea ${ }^{6}$.

\section{Materials and Methods:}

This prospective observational study was carried out in the department of Obstetrics \& Gynaecology, Mymensingh Medical College Hospital, Bangladesh. from July, 2008 to June, 2009. Fifty patients of persistent trophoblastic disease, who were admitted during this period were selected as study population. In this study, there were 49 patients with histologically confirmed molar pregnancies; complete moles in 46 patients and partial moles in 3 patients. One patient had antecedent missed abortion which was not histologically confirmed.

Persistent disease was determined on the basis of the following criteria-

(a) Two or more consecutive weekly increase in beta hCG level.

(b) Plateauing of the beta hCG level for three or more consecutive weeks.

(c) Persistent or recurrent uterine haemorrhage and a persistently detectable beta hCG titre.

(d) Clinical or histological evidence of metastasis.

Evaluation of disease was done by thorough clinical examination and a set of investigations including chest radiography, ultrasound scan of abdomen and pelvis and estimation of serum $\beta$ hCG. The four factors under analysis of PTD were age of the patient, clinical 
presentation, gestational age at diagnosis of molar pregnancy and nature of antecedent pregnancy.

Patients with non metastatic trophoblastic disease and low risk metastatic trophoblastic disease were offered single agent chemotherapy with methotrexate and folinic acid rescue in consultation with the oncologist. When $\beta$ hCG response was inadequate, multi-agent chemotherapy was given. Complications of chemotherapy were also observed and supportive treatment was given.

Sampling Method was Purposive non randomized method. A structured questionnaire was developed addressing all the variables of interest which was finalized following pre-testing.

Data was processed using software SPSS (Statistical Package for Social Science).

\section{Results:}

A total of 50 subjects of persistant trophoblastic disease were included in the present study. Subjects were categorized into different groups, their frequencies with percentages were calculated, significance levels and mean \pm SD were determined by chi square test and one sample "t" test with the help of computer windows SPSS.

Figure-l: Antecedent Pregnancy ( $n=50)$ : 49(98\%) of them were due to post molar trophoblastic disease and $01(02 \%)$ due to missed abortion (Fig I). Among the post - molar trophoblastic disease $46(92 \%)$ due to complete mole and $3(06 \%)$ due to partial mole. Complete mole was statistically significant $(\mathrm{p}=0.001)$.

Table-I: Maternal age of the post trophoblastic subjects $(n=50)$ : Maternal age <20 yrs, $20-30 \mathrm{yrs}, 31-40$ yrs and $>40$ yrs were 01(2.04\%), 28(57.58\%), $14(28.58 \%)$ and $06(12.24 \%)$ respectively of the post molar trophoblastic subjects where 20-30 yrs age group were significantly higher.

Table-I

Maternal age of the post trophoblastic subjects $(n=50)$

\begin{tabular}{lccc}
\hline Years & No. of subjects & Percent (\%) & P value \\
\hline$<20$ & 01 & 2.04 & $<0.001$ \\
$20-30$ & 28 & 57.14 & \\
$31-40$ & 14 & 28.58 & \\
$>40$ & 06 & 12.24 & \\
\hline Total & 49 & 100 & \\
\hline
\end{tabular}

Mean \pm SD

$31.35 \pm 7.25$

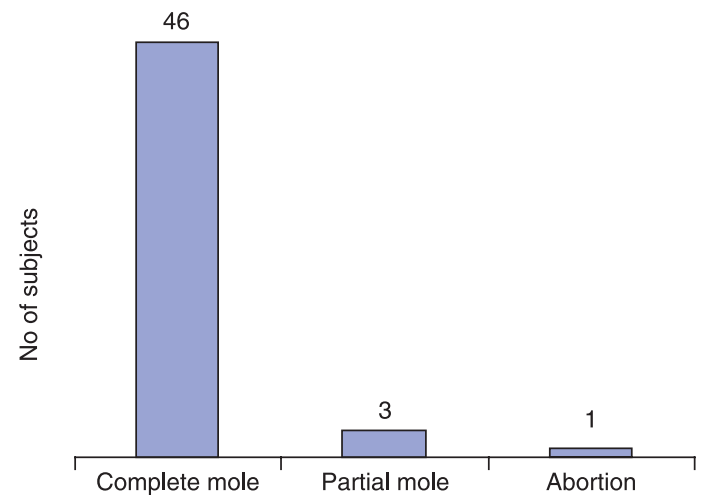

Fig.-1: Antecedent Pregnancy $(n=50)$

Figure-2: Association between past gestational size with the post- molar trophoblastic disease $(n=50)$ : Gestational size <16 wks, $16-20$ wks, and $>20$ wks were 19(38.78\%), 24(48.98\%) and 06(12.24\%) respectively in the post molar trophoblastic subjects. 16-20 wks group were significantly higher.

Fig.-3: Associated theca lutein cyst of the post trophoblastic subjects $(n=50)$ : Associated theca lutein cyst were present in $28(57.14 \%$ ) but absent in $21(42.86 \%)$ in the presentation of trophoblastic subjects which was not statistically significant.

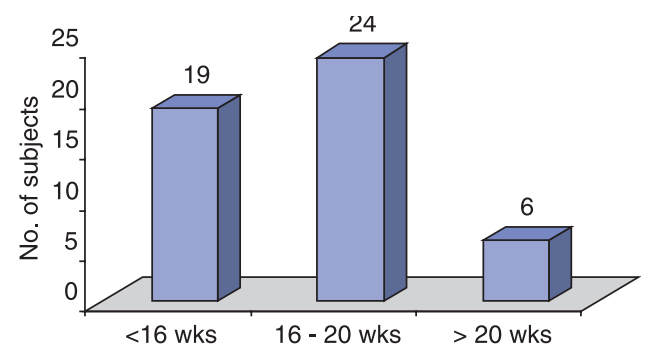

Fig.-2: Association between past gestational Size with the post- molar trophoblastic disease $(n=50)$

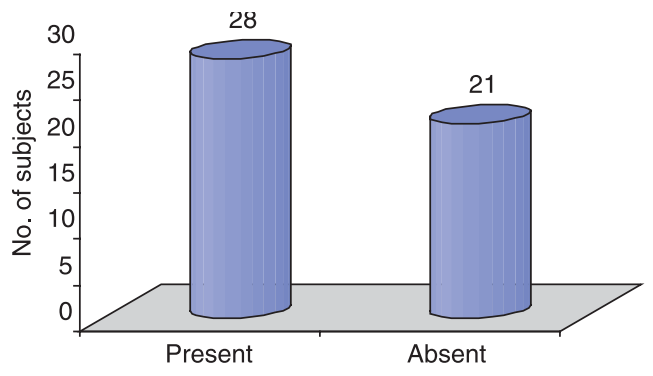

Fig.-3: Associated theca lutein cyst of the post trophoblastic subjects $(n=50)$ 
Figure-IV Menstrual cycle of the study subjects $(n=50)$ : Menstrual cycle were regular in 05(10\%), irregular in 40(80\%) amenorrhea in 04(08\%) and amenorrhea due to surgery in one (02\%) case. Incidence of irregular menstruation was significantly higher.

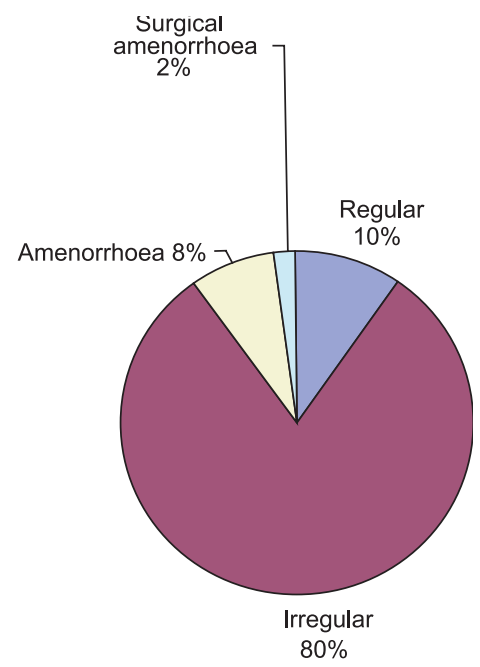

Fig.-4: Menstrual cycle of the study subjects $(n=50)$

Figure-5: Features of distant metastasis of the study subjects $(n=50): 47(94 \%)$ of the study subjects have no features of distant metastasis but 02(04\%) had pulmonary metastasis and one (02\%) case had metastatic nodules in vagina. Non metastatic cases were significantly higher.

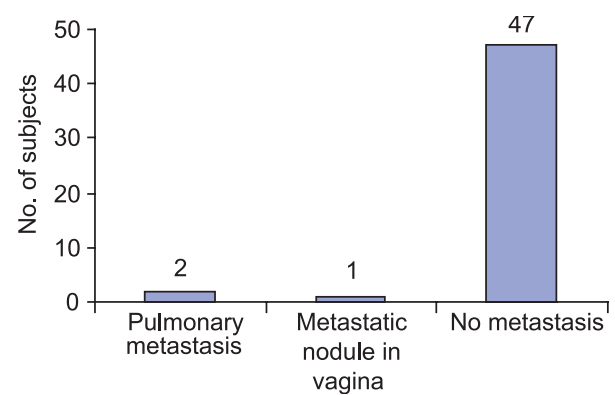

Fig.-5: Features of distant metastasis of the study subjects $(n=50)$

Table-2 Uterine size of the study subjects $(n=50)$ : Uterine size were normal in $17(34 \%), 6-8$ wks in $30(61 \%), 9-12$ wks size in 2(4\%) and $>12$ wks were in $1(2 \%)$ case. Uterine size $6-8$ wks group were significantly higher.

Figure-6: Treatment given to the study subjects $(n=50): 43(86 \%)$ of the study subjects were treated with chemotherapy alone and 07(14\%) had both hysterectomy and chemotherapy.

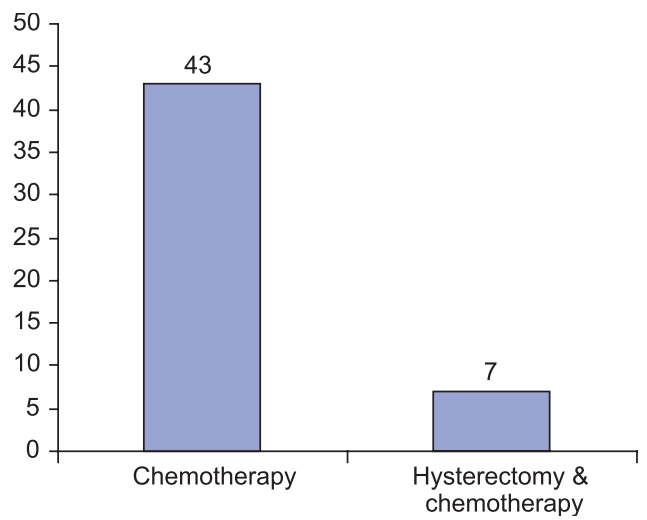

Fig.-6: Treatment given to the study subjects $(n=50)$

Table-II

Uterine size of the study subjects $(n=50)$

\begin{tabular}{lccc}
\hline Size & No. of subjects & Percent (\%) & P value \\
\hline Normal & 17 & 34 & $<0.001$ \\
$6-8$ wks & 29 & 58 & \\
$9-12$ wks & 02 & 04 & \\
$>12$ wks & 01 & 02 & \\
Hysterectomy & 01 & 02 & \\
Total & 50 & 100 & \\
\hline
\end{tabular}

Figure-7: Type of chemotherapy given to the study subjects $(n=50)$ : Single agent chemotherapy were received by $47(94 \%)$ and multiple agent (Etoposide, Methotrexate, Actinomycin D and Cyclophosphamide) by $03(06 \%)$ of the study subjects.

Figure-8: The outcome of the study subjects $(n=50)$ : After chemotherapy, 11(22\%) patients were cured, $38(76 \%)$ had declining â hCG level, and 1(2\%) had static $\beta$ hCG level. Declining $\beta$ HCG level was significantly higher.

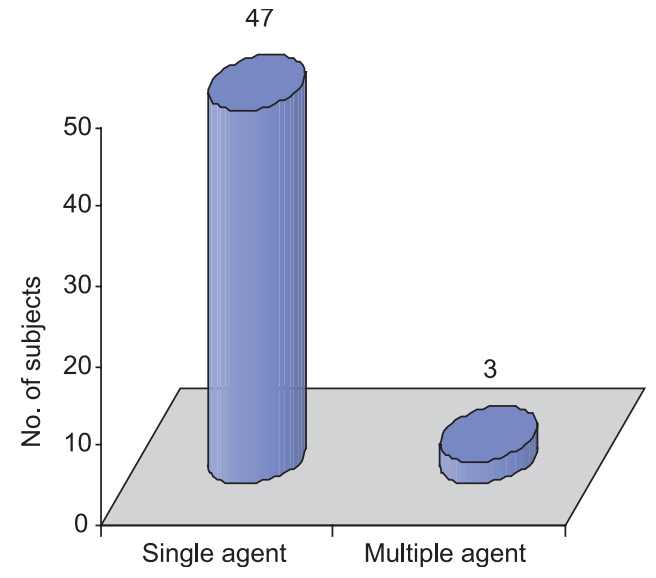

Fig.-7: Type of chemotherapy given to the study subjects $(n=50)$ 


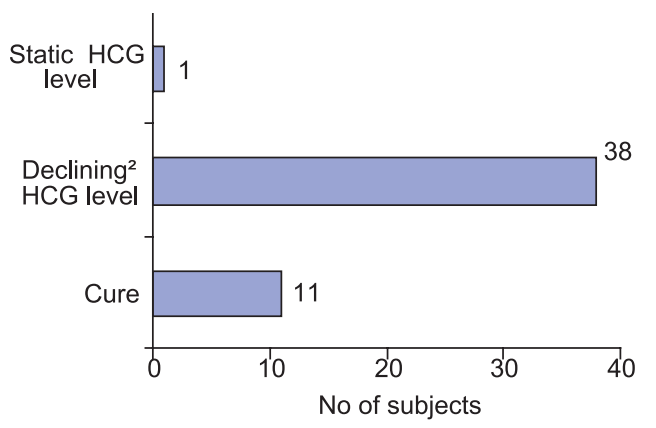

Fig.-8: The outcome of the study subjects $(n=50)$

Figure-9: The complication of chemotherapy of the study subjects ( $n=50): 45(90 \%)$ had no complication of chemotherapy. 02(04\%) had liver dysfunction (Hepatitis and Elevated Transaminases), 02(04\%) had stomatitis and $01(02 \%)$ had both GIT upset and stomatitis.

Figure-10: The effect of chemotherapy on $\beta$ hCG of the study subjects $(n=50)$ : Mean \pm SD of post evacuation $\beta$ hCG was $84551 \pm 39901$, mean \pm SD of $\beta$ hCG levels after $1^{\text {st }}$ dose of chemotherapy was $19854 \pm 14808$ and mean \pm SD of $\beta$ hCG levels after $4^{\text {th }}$ dose of chemotherapy was $84 \pm 50$ (Fig X). The differences were statistically significant.

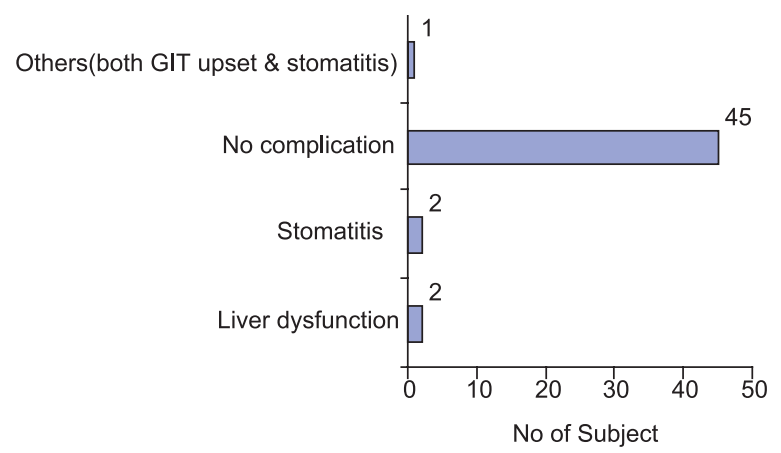

Fig.-9: The complication of chemotherapy of the study subjects $(n=50)$

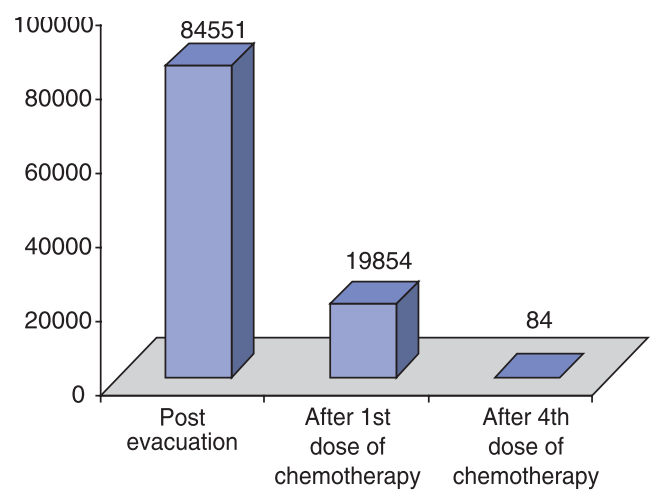

Fig.-10: The effect of chemotherapy of the study subjects $(n=50)$

\section{Discussion:}

Risk factors of post molar trophoblastic disease In the present study, among 49 patients of PTD, 28 (57.14\%) were in 20-30 yrs of age group statistically significant $(p<0.001), 14(28.58 \%)$ in $31-40$ yrs of age and only $7(14.28 \%)$ were in extremes of age. Mean age was $31.35 \mathrm{yr}$. Similar findings were reported in Australia and in New England Trophoblastic Disease Centre (1990-2003) where patient's extreme age (below 20 yrs or above 35 yrs) were not found to be strongly associated with risk of persistent disease ${ }^{7}$, 8 . In another study in India, $47.47 \%$ patients were in between 20 and $25,28 \%$ were less than 20 years of age and $24 \%$ were in $>25$ years $^{9}$. In Philippines, one study reported $44 \%$ of cases in between $25-34$ years and least number of cases in extremes of age ${ }^{10}$.

In this study, 28 (57.14\%) patients had associated theca lutein cyst. But it was statistically in significant. But Tchan Kyu Park found the presence of theca lutein cyst as a significant risk factor ${ }^{4}$.

\section{Antecedent pregnancy}

In this study, among 50 patients of PTD, the antecedent pregnancy was molar in 49 (98\%) and missed abortion in one (2\%) case. Among PTD, 46 $(92 \%)$ were due to complete mole and $3(6 \%)$ due to partial mole.

In Pakistan one study reported among the persistent trophoblastic disease, in $40 \%$ cases antecedent pregnancy was hydatidiform mole, and while $60 \%$ cases it was spontaneous abortion ${ }^{11}$. In Charing Cross Hospital 602 patients treated between 1985 to 1994, there were 100 cases (16.6\%) where the antecedent pregnancy was either a non-molar abortion or a live birth, rest of the cases were molar pregnancy $^{12}$.

\section{Clinical Presentation}

In the present study, 40(80\%) patients presented with irregular pervaginal bleeding which was statistically significant. $(p<0.001), 4(08 \%)$ presented with amenorrhoea and $3(06 \%)$ presented with metastasis, $32(64 \%)$ presented with uterine size more than normal, 28(56\%) with tubo-ovarian mass.

In one study in India 20 cases with GTD were presented with abnormal vaginal bleeding ${ }^{13}$.

In a study in Charring cross hospital, among 602 patients between 1985-1994, approximately two-third $(66.67 \%)$ presented with varying degrees of vaginal 
bleeding but in one-quarter (25\%) of these patients the primary complaint was associated with symptoms from metastatic disease involving lung, brain or abdomen ${ }^{12}$.

\section{Outcome of chemotherapy in persistent trophoblastic disease}

In the present study $43(86 \%)$ patients were treated with chemotherapy and $7(14 \%)$ patients were treated with hysterectomy followed by chemotherapy. All patients started with single agent methotrexate but subsequently $94 \%$ patients responded to single agent and $6 \%$ needed salvage therapy with multiple agent chemotherapy (EMA-CO). After 4 cycles of chemotherapy $22 \%$ patients cured completely, $76 \%$ had declining $\beta$ hCG level and $2 \%$ had static $\beta$ hCG level. Overall remission was $98 \%$. Mean \pm SD of â hCG levels after $1^{\text {st }}$ dose of chemotherapy was $19854 \pm 14808$ and after $4^{\text {th }}$ dose of chemotherapy was $84 \pm 50$ (Table IX and Fig X). The differences were statistically significant.

In India one study showed almost $90 \%$ patient cured with methotrexate (MTX), 2.2\% cases were treated with multiple agent chemotherapy. Case fatality was $2.02 \%{ }^{11}$. Another study showed $92.9 \%$ remission rate of low risk GTN with MTX. Rest of the cases required multiagent chemotherapy ${ }^{14}$.

In Manila, one study reported sustained remission rate $98.5 \%$ with chemotherapy for non metastatic GTN and LRM GTN (Low risk metastatic GTN) ${ }^{12}$ while Wong reported $91.5 \%$ cure rate with $\mathrm{MTX}^{15}$.

In United Kingdom, one study showed $94.4 \%$ cure rate of LRMGTN with MTX while $5.55 \%$ required salvage chemotherapy ${ }^{16}$.

Chemotherapy complication: The toxicity of chemotherapy has been reported to occur in $16.9 \%$ to $70.2 \%$ of the cases. Some deaths due to drug toxicity has been reported. In Korea one study showed chemotherapy was well tolerated without serious toxicities ${ }^{13}$. Hepatotoxicity was observed frequently with MTX, CVF regimen. Gastro intestinal symptoms mainly nausea, vomiting occurred frequently when actinomycin D was given. Haematological toxicity was transient and mild. Alopecia was minimal and reversible. Stomatitis was also mild and transitory.

In the present study, complications of chemotherapy were found only in $10 \%$ of patients. Among them $4 \%$ had liver dysfunction, 4\%had stomatitis and $2 \%$ had both gastrointestinal symptoms and stomatitis.

In Pakistan one study showed after administration of EMA-CO therapy, 100\% patient developed nausea, vomiting, anorexia; $60 \%$ patient developed alopecia and $20 \%$ patient developed bone marrow depression. ${ }^{11}$

In United Kingdom one study showed 25\% patient developed serosal symptoms with low dose MTX and folinic acid regimen, among them pleurisy was the commonest complaint. ${ }^{17}$ On the other hand Wong reported no significant side effects except Steven Johnson Syndrome in one case ${ }^{15}$.

\section{Conclusion:}

Most common presenting complaint of PTD was irregular per vaginal bleeding. Serial $\beta$ hCG levels remain the best monitor for following up a molar pregnancy and for determining response to treatment.

Methotrexate infusion therapy described in this study is effective in the treatment of non metastatic GTD and low risk metastatic GTD. Neither severe complication nor death occurred due to toxicity.

\section{References:}

1. Gerulath A H Clinical Practice Guideline. No. 114 May 2002.

2. Khairunnisa N, Gulfareen H, Nizamuddin M and Ambreen H. Gestational trophoblastic disease: experience at Nawabshah hospital. J Ayub Med Coll Abbottabad 2009; 21(1): 94-7.

3. Nienke ET, Fred CG, Sweep J, Charles PT, Schijf $L$ and Thomas MG. Diagnosis of hydatidiform mole and persistent trophoblastic disease: Diagnostic accuracy of total hCG,free Hcg alfa and beta subunits and their ratios. European Journal of Endocrinology 2005; 153: 565-575.

4. Tchan KP, Soo NK and Sung KL: Analysis of risk factors for postmolar trophoblastic disease :Categorization of risk factors and effect of prophylactic chemotherapy. Yonsei Medical Journal 1996; 37: 412-419.

5. Herbert D. $11^{\text {th }}$ postgraduate course for training in reproductive medicine and reproductive biology. Geneva Foundation for Medical Education and Research. September 4, 2008. 
6. Jeffcoate's principales of gynaecology, 7th edition, jaypee Brothers Medical Publishers 2008; 169-171.

7. Soo KK, David B, Mukhtier S, Wai LY and Lee $\mathrm{T}$. Analysis of risk factors for persistent gestational trophoblastic disease. Australian and Newzealand J of obstetrics and gynaecology 2009; 49: 657-659.

8. Feltmate CM, Growdon WB, Wolfberg AJ and Goldstein DP. Clinical characteristics of persistent gestational trophoblastic neoplasia after partial hydatidiform molar pregnancy. $J$ Reprod Med. 2006; 51(11): 902-6.

9. Shakuntala C and Ambreen Q. Gestational trophoblastic neoplasms with special reference to invasive mole. Obstet Gynecol India March/ April 2007; 57(2): 124-127.

10. Maria S F and Samadan C. Efficacy of methotrexate as primary single agent therapy for non-metastatic and low-risk metastatic gestational trophoblastic neoplasia at the University of the Philippines - Philippine General Hospital. Cancer therapy 2008; 6: 611-616.

11. Izhar R and Aziz N. Prognosis of gestational choriocarcinoma at Khyber Teaching Hospital Peshawar. J Ayub med Coll Abbottabad. 2003; 15(2): 45-8.
12. Newlands ES and Berkowitz RS. Management of persistent gestational trophoblastic disease and gestational trophoblastic tumours. J Reprod Med. 1997; 143-56.

13. Naoki T, Tsuyoshi I, Yoshiko K and Hideyuki U. Gestational trophoblastic disease in women aged 50 or more. Gynecologic Oncology, 1985; 20(1): 53-61.

14. Sekharan PK. Gestational Trophoblastic Disease; Review Article J Obstet Gynecol India July/August 2008; 58(4): 299-307

15. Wong LC, Ngan HY, Cheng DK and Ng TY. Methotrexate infusion in low-risk gestational trophoblastic disease. Am J Obstet Gynecol 2000; 183: 1579-1582.

16. Gillespie AM, Kumar S and Hancock BW. Treatment of persistent trophoblastic disease later than 6 months after diagnosis of molar pregnancy. Br J Cancer 2000; 82(8): 13931395.

17. Sharma S, Jagdev S, Coleman RE, Hancock BW, and Lorigan PC. Serosal complications of single agent low dose methotrexate used in gestational trophoblastic diseases: first reported case of methotrexate induced peritonitis. $\mathrm{Br} \mathrm{J}$ Cancer Nov 1999; 81(6): 1037-1041. 